\title{
Cognitive Impairment and its Correlates in Chronic Kidney Disease Patients Undergoing Haemodialysis
}

\author{
Shijo John Joseph ${ }^{1}$, Samrat Singh Bhandari², Sanjiba Dutta ${ }^{3}$ \\ ${ }^{1}$ Senior Resident, Department of Psychiatry, Sikkim Manipal Institute of Medical Sciences, Sikkim Manipal University, \\ Gangtok, Sikkim, India. ${ }^{2}$ Associate Professor, Department of Psychiatry, Sikkim Manipal Institute of Medical Sciences, \\ Sikkim Manipal University, Gangtok, Sikkim, India. ${ }^{3}$ Professor and HOD, Department of Psychiatry, Sikkim Manipal \\ Institute of Medical Sciences, Sikkim Manipal University, Gangtok, Sikkim, India.
}

\section{ABSTRACT}

\section{BACKGROUND}

Cognitive Impairment (CI) has been found to be quite common amongst patients with Chronic Kidney Disease (CKD) undergoing haemodialysis (HD). The presence of these deficits could affect patient's adherence to diet regimens, treatment and also reduce their Quality of Life. The presence of depression in such patients can further lead to CI. We wanted to assess the prevalence of CI in CKD patients undergoing haemodialysis, socio-demographic and patient related variables affecting $\mathrm{CI}$, and also the relationship between depression and cognition.

\section{METHODS}

Fifty patients undergoing haemodialysis from two dialysis units were assessed. The Montreal Cognitive Assessment (MoCA) scale and Patient Health Questionnaire-9 (PHQ-9) were administered to patients. Descriptive analysis was done for the sociodemographic and clinical variables. Chi square test was used to find the association between the categorical data. Kruskal-Wallis test was used to determine the association between categorical and quantitative variables.

\section{RESULTS}

Mean age of patient was $50.32( \pm 12.4)$ years. Mean duration of dialysis was 18.8 $( \pm 15.11)$ months. The prevalence of depression in the patients was $42 \%$. Cognitive impairment was present in $44 \%$ of the patients. There was a significant relationship between education level and recall $\left(\chi^{2}=31.7, \mathrm{df}=12, \mathrm{p}=.002\right)$ as well as orientation $\left(\chi^{2}=29.78, \mathrm{df}=8, \mathrm{p}=.000\right)$ domains of cognition. Also, there was a significant relationship between socio-economic status and global cognition score $\left(\chi^{2}=81.13\right.$, $\mathrm{df}=48, \mathrm{p}=.002$ ). There was a negative correlation between duration of dialysis and cognition. Significant relationships were found between depression and various cognitive domains.

\section{CONCLUSIONS}

The prevalence of $\mathrm{CI}$ in haemodialysis patients is high. It is also affected by factors such as education level, socio-economic status, duration of dialysis and presence of depression. Insight into $\mathrm{CI}$ is essential for its early identification during the course of illness, so that patient precise treatment decisions can be made.

\section{KEY WORDS}

Renal Dialysis, Cognitive Dysfunction, Depressive Disorder, Patient Health Questionnaire

\author{
Corresponding Author: \\ Dr. Shijo John Joseph, \\ Department of Psychiatry, \\ Sikkim Manipal Institution of Medical \\ Sciences, Sikkim Manipal University, \\ $5^{\text {th }}$ Mile, Tadong, Gangtok-737102, \\ Sikkim, India. \\ E-mail: shijojj90@gmail.com
}

DOI: $10.14260 /$ jemds/2019/611

Financial or Other Competing Interests: None.

How to Cite This Article:

Joseph SJ, Bhandari SS, Dutta S. Cognitive impairment and its correlates in chronic kidney disease patients undergoing haemodialysis. J. Evolution Med. Dent. Sci. 2019;8(36):2818-2822, DOI: 10.14260/jemds/2019/611

Submission 08-07-2019,

Peer Review 25-08-2019,

Acceptance 31-08-2019,

Published 09-09-2019. 


\section{BACKGROUND}

Cognitive impairment (CI) is an expression which has found abode in neuropsychiatry literature since around three decades. ${ }^{1}$ An individual suffering from CI has difficulty in remembering, novel learning, sustaining attention, or decision making which can negatively affect their daily lives. ${ }^{2}$

Chronic Kidney disease is a major public health issue because of its growing prevalence, high morbidity and mortality. Haemodialysis (HD) is the most common modality of renal replacement in India and it is estimated that about 1,20,000 undergo HD. ${ }^{3}$

CKD patients undergoing $\mathrm{HD}$ are at elevated risk of developing CI with moderate to severe $\mathrm{CI}$ being very common. ${ }^{4}$ CI worsens with decline in kidney functioning. ${ }^{5}$ Vascular risk factors like obesity, ageing, hypertensive disorder and diabetes mellitus, CKD related factors like cerebral hypo perfusion, water imbalance, oxidative damage and elevation of uremic toxins all contribute to CI.6,7 Along with these, intradialytic hypo perfusion also may add to deterioration of cognition. ${ }^{8}$ Tamura MK et al in 2017 found that CKD patients shifting to dialysis had decline in executive function, but, not in case of memory or global cognitive scores. ${ }^{9}$

Unidentified CI may result in poor compliance with recommended diet and fluid intake and also poor adherence to medications and HD itself. Identifying $\mathrm{CI}$ would help in making treatment decisions for the nephrologist through consultation liaison. ${ }^{10} \mathrm{CI}$ is associated with reduced Quality of Life (QoL) especially amongst older patients. The present study is done with the intention of assessing the prevalence of CI in CKD patients undergoing haemodialysis, socio-demographic and patient related variables affecting $\mathrm{CI}$ and also the relationship between depression and cognition.

\section{METHODS}

This study is a hospital based cross-sectional study and the participants were the patients attending HD in Sikkim Manipal Institute of Medical Sciences (SMIMS) and Sir Thodup Namgyal Memorial (STNM) Hospital in Gangtok, Sikkim. The study was conducted from March 2016 to March 2017. The study was approved by the Institutional Ethical Committee (IEC, Reg.no. 360/15-083) of SMIMS. At the time of the study both these hospitals were considered as a single unit by the Medical Council of India Hence, the ethical clearance was considered from a single unit then. The participants were selected by convenience sampling. A total of 50 patients who attended the dialysis units of SMIMS AND STNM hospitals and who were willing to participate in the study were included after a written consent was obtained from them. It was decided that all patients undergoing HD and meeting the inclusion criteria and providing informed consent during the one-year period would be taken up for this study. Letter of consent was presented in Nepali and English.

\section{Inclusion and Exclusion Criteria}

Patients who were 18 years of age or older undergoing HD for at least last 3 months were included in the study. Patients who were delirious, those undergoing peritoneal dialysis, those with severe hearing or visual impairment, those who had a history of psychiatric illness prior to the onset of CKD and those who did not give consent were excluded from the study.

\section{Tools/Instruments Used}

1. Montreal Cognitive Assessment (MoCA)

Cognitive function was assessed using the Montreal Cognitive Assessment (MoCA). It measures cognitive function in diverse areas; visuospatial and executive functions, attention, shortterm memory, language and orientation. The maximum score is 30 and it can be administered in 10 minutes. The cut- off score for Mild Cognitive Impairment (MCI) is 26. It has sensitivity and specificity of $90 \%$ and $87 \%$ respectively in detecting MCI. MoCA has been found to be superior to MiniMental Status Examination (MMSE) with regard to sensitivity to identify MCI.11

\section{Patient Health Questionnaire-9 (PHQ-9)}

PHQ is a 9-item depression module, a self-administered version of the Primary Care evaluation of Mental disorders (PRIME-MD) diagnostic tool screening, diagnosing, and measuring the severity of depression. The sum of scores would give a total score representing the severity of depression. Severity of depression is graded as for PHQ-9 score 1 to 4 , the depression severity is none; PHQ-9 score 5-9, depression severity is mild; for score $10-14$, depression severity is moderate; for score $15-19$, depression severity is moderately severe and for score more than 20 , the depression severity is severe. ${ }^{12}$ The sensitivity and specificity of PHQ-9 was found to be $92 \%$ and $92 \%$ respectively. ${ }^{13}$

\section{Statistical Analysis}

Sociodemographic and clinical variables were portrayed using mean and SD for continuous variables and categorical variables were denoted using percentages. The normality of the distribution was assessed by Shapiro-Wilk test. $\chi^{2}$ test was used to analyze the categorical data except when at least one box displayed an expected frequency of less than 5 , in which Fisher's exact correction was applied. Kruskal-Wallis test and Mann-Whitney U test was used to find the association between categorical and quantitative variables. Spearman's rho test was used to determine the correlation between quantitative variables. Data analysis was performed using IBM Statistical Package for Social Sciences, version 20.0 (SPSS 20.0).

\section{RESULTS}

\section{Patient Characteristics}

In this study 50 patients suffering from CKD, undergoing HD from state of Sikkim in India were investigated. Minimum and maximum ages of the patients undergoing HD were 24 years and 70 years respectively with a mean of 50.32 years and SD of 12.4 years. Duration of dialysis for the patients had a mean of 18.8 months with SD of 15.11 months. Other patient characteristics are shown in Table 1 . The prevalence of depression in the patients was $42 \%$. The depression severity among the patients are shown in Fig. 1. Each colour represents the level of severity of depression; light blue for no/ minimal, 
orange for mild, grey for moderate, yellow for moderately severe and dark blue for severe depression.

\begin{tabular}{|c|c|c|}
\hline \multicolumn{2}{|c|}{ Socio-Demographic Variables } & Patient n (\%) \\
\hline \multirow{2}{*}{ Gender } & Male & $33(66)$ \\
\hline & Female & $17(34)$ \\
\hline \multirow{4}{*}{ Marital status } & Single & $3(6)$ \\
\hline & Married & $42(84)$ \\
\hline & Divorced/separated & $4(8)$ \\
\hline & Widowed & $1(2)$ \\
\hline \multirow{5}{*}{ Education } & Illiterate & $2(4)$ \\
\hline & Primary school & $6(12)$ \\
\hline & Secondary school & $10(20)$ \\
\hline & Senior secondary & $27(54)$ \\
\hline & Graduate & $5(10)$ \\
\hline \multirow{6}{*}{ Occupation } & Unemployed & $23(46)$ \\
\hline & Semi-skilled labour & $1(2)$ \\
\hline & Business & $13(26)$ \\
\hline & Salaried (Pvt.) & $7(14)$ \\
\hline & Salaried (Gov.) & $1(2)$ \\
\hline & Retired & $5(10)$ \\
\hline \multirow{4}{*}{ Religion } & Hindu & $37(74)$ \\
\hline & Christian & $2(4)$ \\
\hline & Buddhist & $10(20)$ \\
\hline & Others & $1(2)$ \\
\hline \multirow{5}{*}{ Socio-Economic status } & Lower & $18(36)$ \\
\hline & Lower-Middle & $21(42)$ \\
\hline & Middle & $4(8)$ \\
\hline & Upper Middle & $6(12)$ \\
\hline & High & $1(2)$ \\
\hline \multicolumn{3}{|c|}{ Table 1. Patient Characteristics } \\
\hline
\end{tabular}

\begin{tabular}{|c|c|c|}
\hline Cognitive Domain & Mean & SD \\
\hline Visuo-Spatial/ Executive & 3.64 & 1.562 \\
\hline Naming & 2.60 & .670 \\
\hline Attention & 4.76 & 1.255 \\
\hline Language & 2.88 & .328 \\
\hline Abstract & 2.00 & $.000^{*}$ \\
\hline Recall & 3.96 & .807 \\
\hline Orientation & 5.58 & .702 \\
\hline Table 2. Scores of Cognitive Domains in MoCA \\
\hline
\end{tabular}

\begin{tabular}{|c|c|c|}
\hline \multirow{2}{*}{ Cognition } & \multicolumn{2}{|c|}{ Duration of Dialysis } \\
\cline { 2 - 3 } & Correlation Coefficient & Sig. (2-Tailed) \\
\hline Visuo-Spatial/ Executive & -0.357 & $0.011^{*}$ \\
\hline Naming & -0.358 & $0.011^{*}$ \\
\hline Attention & -0.448 & $0.001^{*}$ \\
\hline Language & -0.313 & $0.027^{*}$ \\
\hline Recall & -0.345 & $0.014^{*}$ \\
\hline Orientation & -0.373 & $0.008^{*}$ \\
\hline MoCA Total score & -0.411 & $0.003^{*}$ \\
\hline \multicolumn{3}{|c|}{ Table 3. Correlation between Cognitive Domains and } \\
Duration of Dialysis \\
\hline
\end{tabular}

\begin{tabular}{|c|c|c|}
\hline \multirow{2}{*}{ Cognition } & \multicolumn{2}{|c|}{ Depression (PHQ-9) } \\
\hline & Correlation Coefficient & Sig. (2-Tailed) \\
\hline Visuo- spatial/Executive & -0.721 & $0.000^{*}$ \\
\hline Naming & -0.574 & $0.000^{*}$ \\
\hline Attention & -0.624 & $0.000^{*}$ \\
\hline Language & -0.598 & $0.000^{*}$ \\
\hline Recall & -0.532 & $0.000^{*}$ \\
\hline Orientation & -0.480 & $0.000^{*}$ \\
\hline MoCA Total score & -0.706 & $0.000^{*}$ \\
\hline \multicolumn{3}{|c|}{ Table 4. Correlation between Cognitive Domains and Depression } \\
\hline
\end{tabular}

\section{Cognitive Impairment}

Cognitive impairment was seen in $44 \%$ of the patients. The mean and SD of MoCA scores were 25.54 and 4.38 respectively. Means and SDs of various domains of cognition are shown in Table-2.

There was a significant association between education level and recall $\left(\chi^{2}=31.7, \mathrm{df}=12, \mathrm{p}=.002\right)$ as well as orientation $\left(\chi^{2}=29.78, \mathrm{df}=8, \mathrm{p}=.000\right)$ domains of cognition. Also, there was a significant association between socio-economic status and global cognition score $\left(\chi^{2}=81.13, \mathrm{df}=48, \mathrm{p}=.002\right)$. No significant associations were found between cognition other socio-demographic variables. There was a negative correlation between duration of dialysis and scores of cognitive domains and global cognition score as shown in Table-3. Attention was the most affected domain with regards to the duration of dialysis. Significant associations were found between depression and various cognitive domains. A negative correlation was found between cognition and severity of depression as shown in Table-4.

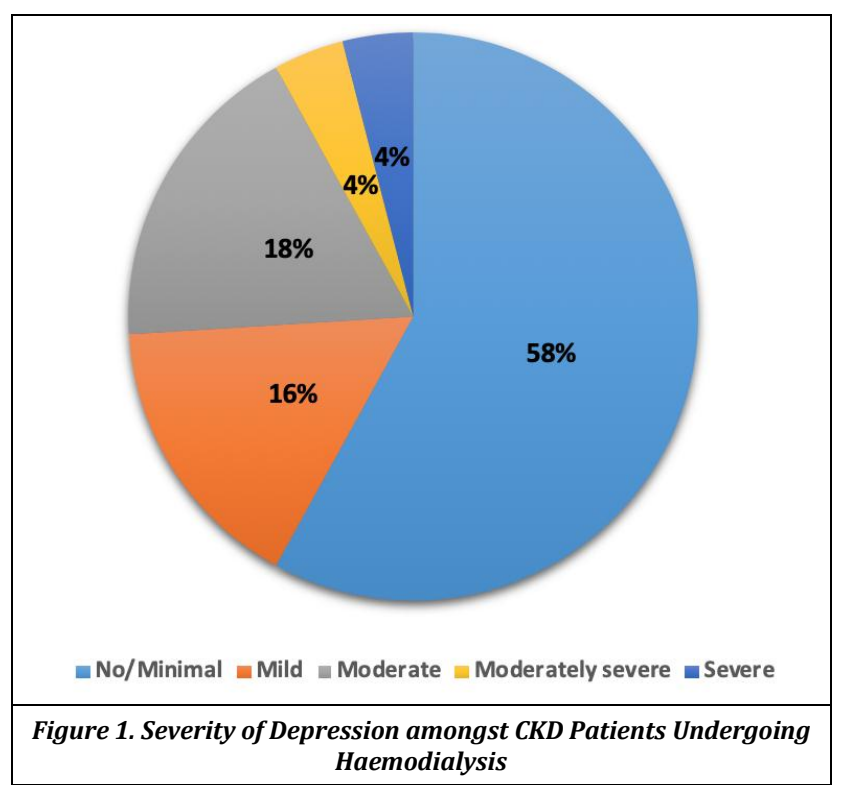

DISCUSSION

The present study included 50 patients suffering from CKD undergoing HD. The mean age of patients was $50.32 \pm 12.4$ years and the mean duration of dialysis was $18.8 \pm 15.11$ months. The prevalence of depression in the patients was $42 \%$. In this research an attempt was made to study cognitive impairment and its correlates in patients with CKD undergoing HD. Using MoCA we found that $44 \%$ of patients were having CI. Some of the studies using MoCA have reported prevalence rates of $41.52 \%$ to $59 \% .^{14-16}$ Other studies which used MMSE found prevalence rates of CI between $8.3 \%$ to $51 \% .{ }^{17-19}$ Research done in this domain around the globe have reported different prevalence rates of CI in HD patients, probably because of different population demographics, sample sizes and measures for assessment. However, the prevalence found in this study is comparable to many of the earlier results.

Fewer years of education and lower levels of education are associated with cognitive dysfunction in these patients as suggested by literature. $17,20,21$ The explanation for this may be association of lower education levels with poor cognitive and functional reserves and deprived lifestyle. Findings in our study are similar except that we had considered levels of education instead of years of education and it was significant in recall and orientation domains. The variation in method of assessment may be a reason for this difference or it may be simply a different finding with respect to this population sample.

It was found in this study that socio economic status had a significant relationship with global cognition score. Previous studies have generally not taken this variable in research.17,21 However, this variable may be significant as socio-economic 
status could affect lifestyle, nutrition and also lead to a delay in diagnosis and treatment of CKD, leading to cognitive impairment. Persons belonging to lower socio-economic status have lower scores of cognition and vice versa.

Our study found that there was a negative correlation between duration of dialysis and cognition. Gesualdo GD et al in 2017 found a weak positive correlation of cognition with dialysis duration. ${ }^{20}$ This may be due to an improvement in medical condition as a result of HD and also the difference in assessment method. Another study by Odagiri et al in 2011 had found that even though that there was no significant relationship between cognition and duration of dialysis. ${ }^{18}$ These findings may because of early diagnosis and management, assessment of patients in the early phase of HD. Variation in the findings of these studies may also suggest that various HD as well as non-dialysis related factors have a role in causing deterioration of cognition. We also found all cognitive domains except abstraction as well as global cognition score, were affected with regard to duration of dialysis; the most affected being domain of attention. Tamura et al in 2010 found decline in executive function domain, which was the only domain prominently measured, however, there was no decline in global cognition score. ${ }^{22}$ The difference in the finding with regard to global cognition score is probably due to the different assessment tool used. In the present study there was a significant association between depression and various domains of cognition as well as global cognition score. Also, a negative correlation was found between depression and cognition scores. Other studies have also found similar decline in cognition with the presence of depression. ${ }^{21,23}$ This can be explained by the effects of symptoms of depression to domains of cognition like executive functioning and processing speed.23 The relationship between cognitive impairment and depression in such patients have not been widely studied. However, a reciprocal relationship between them has been established.

\section{Limitations}

The sample size in the study is small and was done in a single region. We could not determine causal association between variables. Certain factors such as urea, creatinine, creatinine clearance, glomerular filtration rate, other serum/blood parameters could have been included in this research.

\section{CONCLUSIONS}

There is convincingly an unfavourable impact of HD on CKD patients. The high prevalence of $\mathrm{CI}$ in these patients leads to poor QoL and increase in mortality. ${ }^{24}$ Identifying CI early in the course of illness would help the treating team to make patient precise treatment decisions. ${ }^{10}$ The finding in our study unquestionably leads us to the understanding of high prevalence of $\mathrm{CI}$ in these patients. More research of this kind in diverse sociodemographic profiles would help us in better understanding of cognitive impairment and depression of such patients.

\section{ACKNOWLEDGEMENT}

Dr. Dheeraj Khatri, Associate Professor and Chief of the Dialysis Unit, Department of General Medicine, Sikkim Manipal
Institute of Medical Sciences, Gangtok who was a great support during this study.

\section{REFERENCES}

[1] Reisberg B, Ferris SH, de Leon MJ, et al. Stage-specific behavioral, cognitive and in vivo changes in community residing subjects with age-associated memory impairment and primary degenerative dementia of the Alzheimer type. Drug Dev Res 1988;15(2-3):101-14.

[2] https://www.cdc.gov/aging/pdf/cognitive_impairmen t/cogimp_poilicy_final.pdf

[3] Varughese S, Abraham G. Chronic kidney disease in India: a clarion call for change. Clin J Am Soc Nephrol 2018;13(5):802-4.

[4] Murray AM, Tupper DE, Knopman DS, et al. Cognitive impairment in hemodialysis patients is common. Neurology 2006;67(2):216-23.

[5] Davey A, Elias MF, Robbins MA, et al. Decline in renal functioning is associated with longitudinal decline in global cognitive functioning, abstract reasoning and verbal memory. Nephrol Dial Transplant 2013;28(7):1810-9.

[6] Drew DA, Weiner DE. Cognitive impairment in chronic kidney disease: keep vascular disease in mind. Kidney Int 2014;85(3):505-7.

[7] Bugnicourt JM, Godefroy 0, Chillon JM, et al. Cognitive disorders and dementia in CKD: the neglected kidneybrain axis. J Am Soc Nephrol 2013;24(3):353-63.

[8] McIntyre CW, Goldsmith DJ. Ischemic brain injury in hemodialysis patients: which is more dangerous, hypertension or intradialytic hypotension? Kidney Int 2015;87(6):1109-15.

[9] Tamura MK, Vittinghoff E, Hsu CY, et al. Loss of executive function after dialysis initiation in adults with chronic kidney disease. Kidney Int 2017;91(4):948-53.

[10] Tyrrell J, Paturel L, Cadec B, et al. Older patients undergoing dialysis treatment: cognitive functioning, depressive mood and health-related quality of life. Aging Ment Health 2005;9(4):374-9.

[11] Nasreddine ZS, Phillips NA, Bédirian V, et al. The Montreal Cognitive Assessment, MoCA: a brief screening tool for mild cognitive impairment. Journal of the American Geriatrics Society 2005;53(4):695-9.

[12] Kroenke K, Spitzer RL, Williams JB. The PHQ-9: validity of a brief depression severity measure. J Gen Intern Med 2001;16(9):606-13.

[13] Watnick S, Wang PL, Demadura T, et al. Validation of 2 depression screening tools in dialysis patients. Am J Kidney Dis 2005;46(5):919-24.

[14] Saberi A, Monfared A, Sobhani AR, et al. Cognitive impairment and its associated factors in patients undergoing hemodialysis: a cross sectional study. Caspian J Neurol Sci 2017;3(10):118-27.

[15] Wolfgram DF, Sunio L, Vogt E, et al. Haemodynamics during dialysis and cognitive performance. Nephrology (Carlton) 2014;19(12):771-6.

[16] Tiffin-Richards FE, Costa AS, Holschbach B, et al. The Montreal Cognitive Assessment (MoCA) - a sensitive screening instrument for detecting cognitive impairment in chronic hemodialysis patients. PLoS One 2014;9(10):e106700. 
[17] Sithinamsuwan P, Niyasom S, Nidhinandana S, et al. Dementia and depression in end stage renal disease: comparison between hemodialysis and continuous ambulatory peritoneal dialysis. J Med Assoc Thai 2005;88(Suppl 3):S141-S7.

[18] Odagiri G, Sugawara N, Kikuchi A, et al. Cognitive function among hemodialysis patients in Japan. Ann Gen Psychiatry 2011;10:20.

[19] Bossola M, Laudisio A, Antocicco M, et al. Cognitive performance is associated with left ventricular function in older chronic hemodialysis patients: result of a pilot study. Aging Clin Exp Res 2014;26(4):445-51.

[20] Gesualdo GD, Duarte JG, Zazzetta MS, et al. Cognitive impairment of patients with chronic renal disease on hemodialysis and its relationship with sociodemographic and clinical characteristics. Dement Neuropsychol 2017;11(3):221-6.
[21] Jung S, Lee YK, Choi SR, et al. Relationship between cognitive impairment and depression in dialysis patients. Yonsei Med J 2013;54(6):1447-53.

[22] Tamura MK, Larive B, Unruh ML, et al. Frequent Hemodialysis Network Trial Group. Prevalence and correlates of cognitive impairment in hemodialysis patients: the Frequent Hemodialysis Network trials. Clin J Am Soc Nephrol 2010;5(8):1429-38.

[23] Agganis BT, Weiner DE, Giang LM, et al. Depression and cognitive function in maintenance hemodialysis patients. Am J Kidney Dis 2010;56(4):704-12.

[24] Griva K, Stygall J, Hankins $M$, et al. Cognitive impairment and 7-year mortality in dialysis patients. American Journal of Kidney Diseases 2010;56(4):693703. 